\title{
Peer ReViewers • Réviseurs
}

The Canadian Journal of Emergency Medicine recognizes and thanks the following peer reviewers, who have volunteered their time, knowledge and effort to CJEM. Peer review is essential to a medical journal's credibility and success.

This is a list of peer reviewers who helped us in 2006. We apologize in advance if we have overlooked you, but please let us know so we can acknowledge you in a future issue.

Le Journal canadien de la médecine d'urgence tient à remercier les pairs qui ont donné généreusement leur temps, leur savoir et leur efforts à la révision d'articles pour le journal. La révision par les pairs est une composante essentielle à la crédibilité et au succès d'une revue médicale.

Voici la liste des pairs qui nous ont aidés pendant l'année 2006. Nous nous excusons à l'avance si nous avons omis votre nom. Le cas échéant, veuillez nous en aviser afin que nous puissions corriger notre erreur dans un prochain numéro.

A

Stacey Ackroyd

Andrew Affleck

David Agulnik

Samina Ali

Gary Andolfatto

Bryan $\mathrm{Au}$

\section{B}

Mary Ann Badali

Glen Bandiera

Randall Berlin

Ross Berringer

Michael Beug

R.H. Boone

Bjug Borgundvaag

Gary Bota

Robert Brison

Ross Brown

Mike Brzozowski

Jonnathan Busko

C

Ed Cain

Sam Campbell

Christopher Carpenter

Dan Cass

Deepak Chandwani

Eddie Chang

Jim Christenson

Anthony Crocco

Valerie Cunningham

Gil Curry

Sarah Curtis

\section{D}

Patricia Daly

Robert Dart

Soraya Davoudi

Brian Deady

Valerie De Maio

Chris Denny

Chris DeWitt

Alba DiCenso

Ed Dillon

Ken Dittrich

Alan Drummond

Robert Drummond

\section{E}

Mary Eisenhaur

Marcel Émond

Michael Ertel

Chris Evans
Lisa Evered
F
Jerome Fan
Ken Farion
Louis Francescutti
Jeff Freeman
John Froh
G
Roger Galbraith
Annie Gareau
Yuri Gilhotra
Ken Gin
Jim Goulding
Eric Grafstein
Tim Graham
Vincent Grant
Robert Green
Dianne Groll
H
L
Joe Haegert
Tammy Hall
Vincent Hanlon
Devin Harris
Dave Harrison
William Henderson
Anna Karwowsiberte
Anne-Maree Kelly
John Kendall
Anthony Herd
Michael D. Hill
Corinne Hohl
Brian Holroyd
Daniel Howes
Michael Howlett
Marilyn Innes
Dean Johnston
Gord Jones
Gary Joubert

Eddy Lang
Éric Laviolette
Connie LeBlanc
Eric Letovsky
Joel Lexchin
Jason Lord
Adam Lund
M
Duncan Mackey
David Mann
David Maxwell
Gary Mazowita
Russel McDonald
J. Gordon McInnes
Douglas McKnight
Bruce McLeod
Ron McMillan
Iain McPhail
Kelly Millar
Bruce Minnes
Ameer Mody
Kieran Moore
Don Morel
Leigh Morris
Nancy Murphy
Michael Murray
David Mutrie

N-O
Joe Nemeth
Gina Neto
Cheri Nijssen-Jordan
Howard Ovens

P

Wes Palatnick

Linda Papa

Eleena Pearson

Jeff Perry

David Petrie

Jeff Plant

Julien Poitras

Greg Powell

Simon Pulfrey

R

Jock Reid

David Rhine

Ian Rigby

Brian Rowe
S

Gregg Scheirer

Michael Schull

William Sevcik

Jonathan Sherbino

Gil Shlamovitz

Michael Shuster

Douglas Sinclair

Richard Slavik

Victoria Slavik

Carolyn Snyder

Steve Socransky

Julie Spence

Carol Spooner

Claud Stang

Brian Steinhart

Rob Stenstrom

Charles Sun

T

Anthony Taddei

Jack Taunton

Brett Taylor

Milton Tenenbein

James Thompson

Margaret Thompson

Knox Todd

U-V

Suneel Upadhye

Alain Vadeboncoeur

Christian Vaillancourt

Jonathan Valente

Les Vertesi

Don Voaklander

W

Ian Walker

Karen Wanger

Michael Wansbrough

John Ward

David Warren

Mary-Lynn Watson

Nancy Weber

Michelle Welsford

Sandy Whitehouse

Ray Wiss

Jonathan Witt

Michael Witt

Michael Woo

Vic Wood

Karen Woolfrey 